\title{
Dust heating
}

\section{Photon absorption versus electron collisions}

\author{
M. Bocchio ${ }^{1}$, A. P. Jones ${ }^{1}$, L. Verstraete ${ }^{1}$, E. M. Xilouris ${ }^{2}$, E. R. Micelotta ${ }^{3}$, and S. Bianchi ${ }^{4}$ \\ ${ }^{1}$ Institut d'Astrophysique Spatiale (IAS), UMR 8617, CNRS/Université Paris-Sud, 91405 Orsay, France \\ e-mail: marco.bocchio@ias.u-psud.fr \\ 2 Institute for Astronomy, Astrophysics, Space Applications \& Remote Sensing, National Observatory of Athens, \\ P. Penteli, 15236 Athens, Greece \\ 3 Department of Physics and Astronomy, University of Western Ontario, London, Ontario N6A 3K7, Canada \\ ${ }^{4}$ INAF - Osservatorio Astrofisico di Arcetri, Largo E. Fermi 5, 50125 Florence, Italy
}

Received 7 January 2013 / Accepted 18 June 2013

\section{ABSTRACT}

\begin{abstract}
Aims. We investigate and quantify the effects of the electron collisional heating of dust in a hot gas and compare this with photon heating by the interstellar radiation field.

Methods. We compare the rate of energy absorption by dust due to electron collisional and photon heating as a function of the physical conditions of the gas and the ambient radiation field. We calculate the resulting dust spectral energy distributions (SEDs) for different environments.

Results. We find that electron collisions and grain charging effects in a hot gas $\left(10^{6}-10^{7} \mathrm{~K}\right)$ rapidly destroy small carbonaceous particles and result in a minimum particle size of the order of a few $\mathrm{nm}$. The charging due to the emission of secondary electrons is important and leads to high electric potentials, which quickly destroy the small grains by field ion emission. In the case of weak interstellar radiation fields $\left(G_{0} \sim 0.1\right)$, electron collisional heating can be the dominant heating process and therefore makes an important contribution to the dust thermal emission.

Conclusions. Collisions of electrons with dust grains, in a hot gas, lead to important changes in the dust SED, as a result of their high energy input. We find that grain charge effects and accompanying erosion need to be taken into account in the calculation of the dust SED. The power absorbed by the dust as a result of electron collisions in a hot tenuous gas can be larger than that due to photon absorption in the intergalactic medium close to a galaxy where the radiation field is weak $\left(G_{0} \lesssim 0.1\right)$.
\end{abstract}

Key words. dust, extinction - radiation mechanisms: thermal - galaxies: clusters: intracluster medium - radiative transfer galaxies: individual: NGC 891

\section{Introduction}

Observations of ram-pressure stripped galaxies in the Virgo Cluster have shown a correlation and an asymmetry of dust and gas profiles with respect to the galactic plane (e.g. Cortese et al. 2010). Dust and gas pushed outside the boundaries of a galaxy will encounter a different, more tenuous and much warmer environment than the interstellar medium (ISM): the intergalactic medium (IGM). Since the IGM is a very hot and tenuous medium $\left(T \approx 10^{5}-10^{8} \mathrm{~K}, n_{\mathrm{H}} \approx 10^{-3}-10^{-4} \mathrm{~cm}^{-3}\right.$ ), dust will not only be heated by the photon absorption but also by electron collisions, which could play an important role. Furthermore, there are other environments where electron collisional heating may be important i.e. high- and intermediate-velocity clouds (HVCs and IVCs), galactic fountains and in the hot ionised medium (HIM) or coronal gas in the Milky Way or elliptical galaxies.

However, both of these grain heating processes, i.e. photon absorption and electron collisions, will contribute not only to dust heating but also to its destruction (see Micelotta et al. 2010; Bocchio et al. 2012, for particle erosion in a hot gas). In particular, this would mostly affect the smallest grains in the size distribution.

In this paper, we introduce electron collisional heating into the DustEM code ${ }^{1}$ and compare its effect with that due to

\footnotetext{
1 http://wWw.ias.u-psud.fr/DUSTEM
}

photon absorption in the IGM in order to investigate if dust would be able to emit enough energy to be detected by modern infrared/far-infrared (FIR) telescopes.

\section{The dust model}

In this work we use the Compiègne et al. (2011) dust model. This dust model consists of four types of grains: polycyclic aromatic hydrocarbons (PAHs), small and large hydrogenated amorphous carbon (SamC and LamC) grains and silicate grains (aSil). The PAHs and SamC grains follow a $\log$ normal $(\mathrm{d} n / \operatorname{dlog} a \propto$ $\left.\exp \left[-\frac{\log \left(a / a_{0}\right)^{2}}{2 \sigma^{2}}\right]\right)$ size distribution while the LamC grains and silicate grains follow a power law $\left(\mathrm{d} n / \mathrm{d} a \propto a^{\alpha}\right)$ starting at $a_{\min }$ and with an exponential cut-off of the form $\mathrm{e}^{-\left[\left(a-a_{\mathrm{t}}\right) / a_{\mathrm{c}}\right]^{\gamma}}$ for $a \geq a_{\mathrm{t}}$ (1 otherwise). The parameters that characterize the size distributions are summarized in Table 1 and the corresponding mass distribution is plotted in Fig. 1.

It is clear from Bocchio et al. (2012) that, for small carbonaceous particles $(a \leqslant 5 \mathrm{~nm}$ ), the 2D and 3D effective cross sections are equivalent. This allows us to use a $3 \mathrm{D}$ approach to describe PAH destruction.

\section{Electron collisional heating}

In a fully ionised hot coronal-type gas, be it galactic HIM or nearby IGM, dust is heated by inelastic collisions with the ambient ions and electrons. At a given gas temperature, ions 


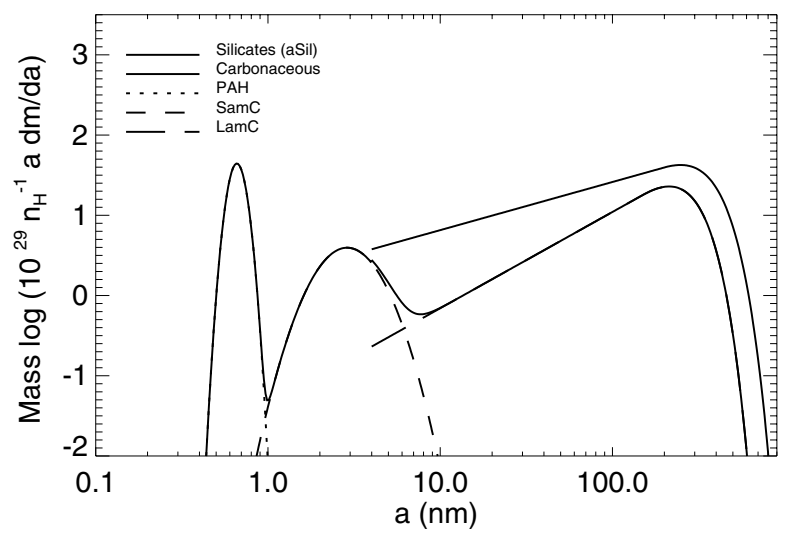

Fig. 1. Mass distributions for the four dust types of the Compiègne et al. (2011) model as a function of the grain radius.

Table 1. Size distribution parameters for the Compiègne et al. (2011) dust model (see text for details).

\begin{tabular}{lcccccc}
\hline \hline & $\begin{array}{c}\rho \\
\left(\mathrm{g} \mathrm{cm}^{-3}\right)\end{array}$ & $\begin{array}{c}a_{\min } / a_{\max } \\
(\mathrm{nm})\end{array}$ & $\sigma$ & $\begin{array}{c}a_{0} \\
(\mathrm{~nm})\end{array}$ & $\begin{array}{c}Y \\
\left(M / M_{\mathrm{H}}\right)\end{array}$ \\
\hline PAH & 2.24 & $0.35 / 1.2$ & 0.1 & 0.64 & $7.8 \times 10^{-4}$ \\
SamC & 1.81 & $0.6 / 20$ & 0.35 & 2.0 & & $1.65 \times 10^{-4}$ \\
& & & & & & \\
\hline & $\begin{array}{c}\rho \\
\left(\mathrm{g} \mathrm{cm}^{-3}\right)\end{array}$ & $\begin{array}{c}a_{\min } / a_{\max } \\
(\mathrm{nm})\end{array}$ & $\alpha$ & $\begin{array}{c}a_{\mathrm{c}}, a_{\mathrm{t}} \\
(\mathrm{nm})\end{array}$ & $\gamma$ & \\
\hline LamC & 1.81 & $4 / 2000$ & -2.8 & 150 & 2.0 & $1.45 \times 10^{-3}$ \\
aSil & 3.0 & $4 / 2000$ & -3.4 & 200 & 2.0 & $7.8 \times 10^{-3}$ \\
\hline & & & \multicolumn{5}{c}{ TOTAL } & $10.2 \times 10^{-3}$ \\
\hline
\end{tabular}

Notes. $Y$ is the mass abundance per hydrogen for each dust component.

and electrons will have the same energy but, because electrons have a much smaller mass, they will reach higher velocities with respect to the ions. This implies that the electron flux is larger than that of the ions and therefore the major contribution to the collisional heating is given by electrons. The effect of electron collisional heating has already been studied in the case of dust in a low density, X-ray emitting plasma by Dwek $(1986,1987)$ who calculated the equilibrium temperature of very small grains heated only by collisions with the ambient electrons of a hot gas $\left(T \approx 10^{7} \mathrm{~K}\right)$. He also calculated the dust-temperature distribution which is a key factor in determining the dust spectral energy distribution (SED).

Following Dwek (1986), the power absorbed per grain (be it a carbon or a silicate grain) due to electron collisions is given by:

$H=\pi a^{2} n_{\mathrm{e}} \int_{0}^{\infty} f_{\mathrm{M}}(E) v(E) \tilde{\sigma}(E) E \zeta(E) \mathrm{d} E$

where $a$ is the grain radius, $n_{\mathrm{e}}$ is the electron density (which equals the proton density, $n_{\mathrm{H}}$, since the gas is fully ionised), $f_{\mathrm{M}}(E)$ is the Maxwell-Boltzmann energy distribution, $v(E)$ the velocity of the impinging electron, $E$ its energy, $\tilde{\sigma}(E)$ represents the Coulomb focusing and $\zeta(E)$ the fraction of energy transferred to the target grain. As discussed in Sect. 6, Coulomb focusing has little effect on the grain charge under these conditions and is ignored here.

Dwek (1986) gives an estimate of the transferred energy, $E \zeta(E)$, as a function of the energy of the impinging electron:

$\zeta(E)= \begin{cases}1 & \text { for } E<E_{\mathrm{th}} \\ 1-\left[1-\left(E_{\mathrm{th}} / E\right)^{3 / 2}\right]^{2 / 3} & \text { for } E>E_{\mathrm{th}}\end{cases}$

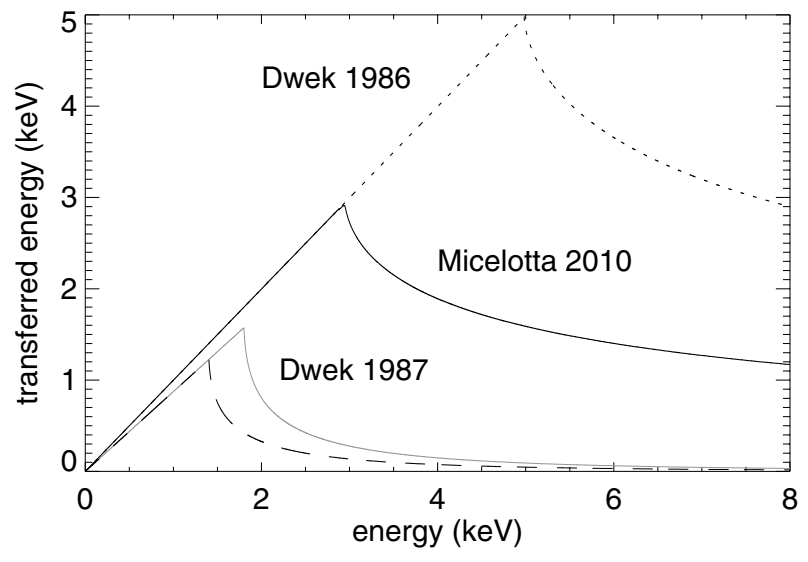

Fig. 2. Transferred energy as a function of the kinetic energy of the impinging electrons for carbonaceous and silicate grains with a radius of $0.1 \mu \mathrm{m}$. Carbonaceous grain (black lines): solid line (Micelotta et al. 2010), dotted line (Dwek 1986), dashed line (Dwek 1987). Silicate grain, grey solid line (Dwek 1987).

Table 2. Analytical fit (to within few \%) to the electron stopping power in solid carbon (Micelotta et al. 2010).

\begin{tabular}{lccc}
\hline \hline \multicolumn{4}{c}{$S(E)=(h \ln (1+a E)) /\left(f E^{g}+b E^{d}+c E^{e}\right)$} \\
\hline$a$ & $b$ & $c$ & $d$ \\
$-4.23 \times 10^{-4}$ & $-3.57 \times 10^{-11}$ & $-3.38 \times 10^{-7}$ & -3.19 \\
\hline$e$ & $f$ & $g$ & $h$ \\
-0.588 & $-2.33 \times 10^{-4}$ & 1.54 & 1.41 \\
\hline
\end{tabular}

Notes. The fit parameters are given to 3 significant digits and $E$ is in $\mathrm{keV}$.

with the threshold energy $E_{\mathrm{th}}(\mathrm{erg})=3.7 \times 10^{-8} a(\mu \mathrm{m})^{2 / 3}$.

Dwek (1987) updates his previous estimate of the transferred energy function in electron collisions. His study is based on the experimental ranges of electrons in all media within the energy range of $20 \mathrm{eV}$ to $1 \mathrm{MeV}$ (Iskef et al. 1983; Berger \& Seltzer 1964). This new estimate of the energy transferred is functionally very similar to the previous estimate but it has a lower threshold. Dwek (1987) does not give a simple expression for the threshold energy. For a $0.1 \mu \mathrm{m}$ carbonaceous grain of density $\rho=1.8 \mathrm{~g} \mathrm{~cm}^{-3}$, following his method, as we can deduce from Fig. 1 by Dwek (1987), the threshold energy equals $E_{\text {th }} \sim 1.4 \mathrm{keV}$ (see Fig. 2).

A more recent approach is given by Micelotta et al. (2010). They derive the electron energy loss (for $E=10 \mathrm{eV}-2 \mathrm{keV}$ ) in carbon from measurements of $\mathrm{d} E / \mathrm{d} x$ for solid carbon (Joy 1995). The data points are well fit by the following function:

$S(E)=\frac{h \ln (1+a E)}{f E^{g}+b E^{d}+c E^{e}}$

where $S(E)$ is the stopping power and $E$ the energy of the impinging electron. The fit parameters are listed in Table 2 . In order to find the transferred energy to a spherical grain due to electron collisions Micelotta et al. (2010) used the following procedure. Since $S(E)=-\mathrm{d} E / \mathrm{d} x$, integrating over the electron path through the grain we find:

$l_{\max }=\int_{x_{0}}^{x_{1}} \mathrm{~d} x=-\int_{E_{0}}^{E_{1}} \frac{\mathrm{d} E}{S(E)}=F\left(E_{0}\right)-F\left(E_{1}\right)$,

where $F(E)$ is the path length travelled by an electron with an energy $E, E_{0}$ the energy of the impinging electron, $E_{1}$ the energy 
of the electron after having travelled through the grain and $l_{\max }$ is the maximum path length through the grain. The value of $l_{\max }$ is of course limited to the maximum path length through the grain if the electrons are not stopped within the grain. In that case, the average path length traversed through a spherical grain is $4 \mathrm{a} / 3$. Therefore, for a given $E_{0}$ we can derive $E_{1}$ and we define the transferred energy as:

$E_{\text {tr }}=E_{0}-E_{1}$.

In Fig. 2 we show the transferred energy as a function of the energy of the impinging electron. Black lines refer to the transferred energy into carbonaceous grains whilst the grey line refers to silicate grains. For these calculations we assume a carbon grain of density $1.8 \mathrm{~g} \mathrm{~cm}^{-3}$ and a radius of $0.1 \mu \mathrm{m}$ and a silicate grain of density $3 \mathrm{~g} \mathrm{~cm}^{-3}$ and the same radius. We compare the three approaches described for the transferred energy into carbonaceous grains. The dotted line shows the approach of Dwek (1986), the dashed line the approach of Dwek (1987) and the black solid line the approach of Micelotta et al. (2010). We notice that, even if the methods used to calculate the transferred energy are quite different from one another, the functional behavior is very similar. The difference between the transferred energy function for carbonaceous and silicate grains adopted by Dwek (1987) is simply due to the different grain densities used there. In our model we use the transferred energy function obtained by Micelotta et al. (2010) for carbonaceous grains (black solid line) and, since there are no more recent estimates, we use that obtained by Dwek (1987) for silicate grains (grey solid line).

\section{Photon heating}

In regions where a radiation field is present, grains can be heated by photon absorption. In this case the power absorbed per grain $\left(H_{\gamma}\right)$ is given by:

$H_{\gamma}=\pi a^{2} \int_{v_{\min }}^{v_{\max }} Q_{\mathrm{abs}} J_{\nu} \mathrm{d} v$

where $v_{\min }$ and $v_{\max }$ are the minimum and maximum frequencies of the radiation field, $\pi a^{2} Q_{\text {abs }}$ is the absorption cross section and $J_{v}$ the intensity of the radiation field at the given frequency $v$. To quantify the power absorbed in this way we need to estimate the radiation field in the region we want to consider, in this case in the IGM, a few kpc away from the galactic plane.

We focus here on the study of the radiation field in the proximity of spiral galaxies. Edge-on galaxies play an important role in this study since we have experimental data on the radiation field in the optical/near-infrared (NIR) bands around the galactic plane. A good example of an edge-on galaxy is NGC 891 and its radiation field has been widely studied in the literature (e.g. Xilouris et al. 1999; Popescu et al. 2000). We use this galaxy only as an example to have an estimate of the radiation field around a typical spiral galaxy. Xilouris et al. (1999) modeled the distribution of stars and dust in the optical and NIR bands. The stellar distribution that they use is composed of an exponential (both in the radial, $R$, and vertical, $z$, directions) disk and a bulge (which in projection is given by the well-known $R^{1 / 4}$ law, Christensen 1990) described by:

$$
\begin{aligned}
\eta_{0}(R, z)= & L_{\mathrm{s}} \exp \left(-\frac{R}{h_{\mathrm{s}}}-\frac{|z|}{z_{\mathrm{s}}}\right) \\
& +L_{\mathrm{b}} \exp \left(-7.67 B^{1 / 4}\right) B^{-7 / 8}
\end{aligned}
$$

Table 3. Parameters for NGC 891 derived by Xilouris et al. (1999).

\begin{tabular}{lccccc}
\hline \hline Parameter & $B$ & $V$ & $I$ & $J$ & $K$ \\
\hline$L_{\mathrm{s}}\left[\frac{\mathrm{erg}}{\mathrm{spc}^{3} \mathrm{srnm}} \times 10^{28}\right]$ & 2.66 & 3.53 & 3.44 & 6.2 & 1.41 \\
$z_{\mathrm{s}}[\mathrm{kpc}]$ & 0.43 & 0.42 & 0.38 & 0.43 & 0.34 \\
$h_{\mathrm{s}}[\mathrm{kpc}]$ & 5.67 & 5.48 & 4.93 & 3.86 & 3.87 \\
$L_{\mathrm{b}}\left[\frac{\mathrm{erg}}{\mathrm{spc}^{3} \mathrm{srnm}} \times 10^{31}\right]$ & 12.0 & 7.4 & 2.23 & 4.99 & 1.71 \\
$R_{\mathrm{e}}[\mathrm{kpc}]$ & 1.12 & 1.51 & 1.97 & 0.87 & 0.86 \\
$b / a-$ & 0.6 & 0.54 & 0.54 & 0.71 & 0.76 \\
$\tau^{f}-$ & 0.87 & 0.79 & 0.58 & 0.23 & 0.10 \\
$z_{\mathrm{d}}[\mathrm{kpc}]$ & 0.27 & 0.27 & 0.27 & 0.27 & 0.27 \\
$h_{\mathrm{d}}[\mathrm{kpc}]$ & 7.97 & 7.97 & 7.97 & 7.97 & 7.97 \\
\hline
\end{tabular}

Notes. The values of $z_{\mathrm{d}}$ and $h_{\mathrm{d}}$ represent the average values of those found by Xilouris et al. (1999) for the different bands.

where $\eta_{0}$ is the total stellar emissivity (in erg s${ }^{-1} \mathrm{pc}^{-3} \mathrm{sr}^{-1} \mathrm{~nm}^{-1}$ ) at $(R, z), L_{\mathrm{s}}$ and $L_{\mathrm{b}}$ are the normalization constants for the stellar emissivity of the disk and the bulge, $h_{\mathrm{s}}$ and $z_{\mathrm{s}}$ are the stellar scalelength and scaleheight and

$B=\frac{\sqrt{R^{2}+z^{2}(a / b)^{2}}}{R_{\mathrm{e}}}$

with $R_{\mathrm{e}}$ the effective radius of the bulge and $a$ and $b$ the semimajor and semi-minor axes respectively. To model the presence of a dust disk in the galaxy they used an exponential law for the extinction coefficient:

$\kappa_{\lambda}(R, z)=\kappa_{\lambda}(0,0) \exp \left(-\frac{R}{h_{\mathrm{d}}}-\frac{|z|}{z_{\mathrm{d}}}\right)$,

where $\kappa_{\lambda}(R, z)$ is the extinction coefficient (in $\mathrm{kpc}^{-1}$ ) at wavelength $\lambda$ at $(R, z), h_{\mathrm{d}}$ and $z_{\mathrm{d}}$ are the dust scalelength and scaleheight respectively. $\kappa_{\lambda}(0,0)$, the extinction coefficient at the centre of the disk, is related to the optical depth of the galaxy as seen face-on, $\tau_{\lambda}^{f}$ and the scaleheight:

$\tau_{\lambda}^{f}=2 \kappa_{\lambda}(0,0) z_{\mathrm{d}}$

In this work we use the parameters listed in Table 3 (Xilouris et al. 1999).

The same model has been used by Popescu et al. (2000). Starting from the optical-NIR SED, they calculated the FIR SED emitted by the dust distribution and compared this with the observational data from Alton et al. (1998). The observed differences between the results of the model and the data were assumed to be due to the absence of the UV radiation field. They assumed a distribution of young stars with a scaleheight smaller than the dust scaleheight. In fact, in edge-on galaxies, it is very difficult to observe any radiation in the UV because it is absorbed by the dust lane.

To compute the radiation in the UV it is possible to use the relation between SFR and the luminosity at different UV wavelengths, based on population synthesis models (e.g. Fioc \& Rocca-Volmerange 1997). Misiriotis et al. (2004) calculated this relation with the PEGASE population synthesis model of Fioc \& Rocca-Volmerange (1997) and obtained:

$S F R=8.12 \times 10^{-28} L_{\lambda}\left[\mathrm{erg} \mathrm{s}^{-1} \mathrm{~Hz}^{-1}\right]$ at $\lambda=91.2 \mathrm{~nm}$

and

$S F R=1.4 \times 10^{-28} L_{\lambda}\left[\mathrm{erg} \mathrm{s}^{-1} \mathrm{~Hz}^{-1}\right]$ at $\lambda=150 \mathrm{~nm}$ and $\lambda=280 \mathrm{~nm}$. 


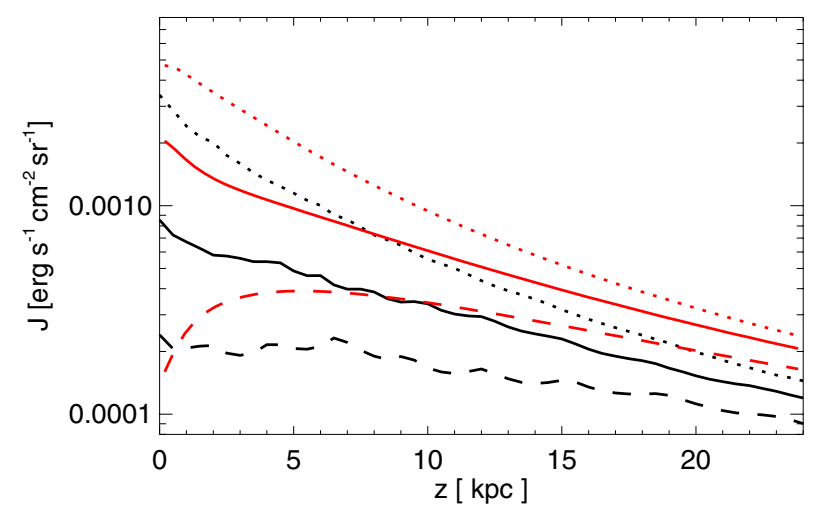

Fig. 3. Integrated radiation field $J\left[\mathrm{erg} \mathrm{s}^{-1} \mathrm{~cm}^{-2} \mathrm{sr}^{-1}\right.$ ] as a function of the distance from the galactic plane for $R=1,2,3 h_{\mathrm{s}}$ (dotted, solid and dashed lines respectively) where $h_{\mathrm{s}}=5.6 \mathrm{kpc}$. Red lines show the radiation field obtained with the TRADING model while black lines correspond to the model described in this paper.

Furthermore, Popescu et al. (2000) assumed that a fraction $y_{\mathrm{UV}}$ of the non-ionising UV is absorbed in star-forming complexes (e.g. HII regions). They fit the FIR SED to the observational data and find a value $S F R=3.5 M_{\odot} / \mathrm{yr}$ and $y_{\mathrm{UV}}=0.28$ for NGC 891 . As per Popescu et al. (2000) we assume that the young stellar population has a scaleheight of $z_{\mathrm{UV}}=90 \mathrm{pc}$ and a scalelength similar to that of the disk in the blue-band (i.e. $h_{\mathrm{UV}}=5.67 \mathrm{kpc}$ ). From the SFR - luminosity relations, using Eq. (7) and assuming $y_{\mathrm{UV}}=0.28$ we calculate the stellar emissivity at wavelengths $\lambda=91.2,150$ and $280 \mathrm{~nm}$ :

$L_{\mathrm{s}}\left[\frac{\mathrm{erg}}{\mathrm{s} \mathrm{pc}^{3} \mathrm{srnm}} \times 10^{29}\right]= \begin{cases}2.45 & \text { for } \lambda=91.2 \mathrm{~nm} \\ 5.25 & \text { for } \lambda=150 \mathrm{~nm} \\ 1.51 & \text { for } \lambda=280 \mathrm{~nm}\end{cases}$

We now need to calculate the radiation field that dust would experience at each point in the galaxy NGC 891. To do this, we use the method described by Dasyra et al. (2005) (see also Misiriotis et al. 2006). Given the parameters of the stellar and dust distributions, we calculate the radiation field at a given point inside the galaxy taking into account both the effects of absorption and multiple scattering by dust and the subsequent re-emission at FIR/submm wavelengths.

We compare our model with the more sophisticated TRADING model (Bianchi 2008; Bianchi \& Xilouris 2011) based on Monte Carlo (MC) simulations. The TRADING model of NGC 891 has been derived from the geometric properties and dust opacities obtained by Xilouris et al. (1999). For the intrinsic stellar spectrum a template for an Sb galaxy was used, scaled to match available NIR/optical/UV fluxes. As per Popescu et al. (2000), an additional dust component was included to fit the observed FIR/submm SED. In the TRADING model, this component is in the form of an exponential disk distribution of spherical (molecular) clouds, heated internally by a UV-dominated radiation field from young stars. In Bianchi \& Xilouris (2011) the model was fine-tuned to match both the global SED and the spatial distribution of the dust emission observed by the Herschel Space Observatory.

Figure 3 presents the radiation field integrated over the wavelengths $J$ [erg s $\mathrm{em}^{-1} \mathrm{~cm}^{-1} \mathrm{sr}^{-1}$ as a function of the distance from the galactic plane, $z$, for a radial distance $R=1,2,3 h_{\mathrm{s}}$ (dotted, solid and dashed lines respectively) where $h_{\mathrm{s}}=5.6 \mathrm{kpc}$. Red lines show the TRADING model results, black lines show the model described in this paper. We notice a different behaviour

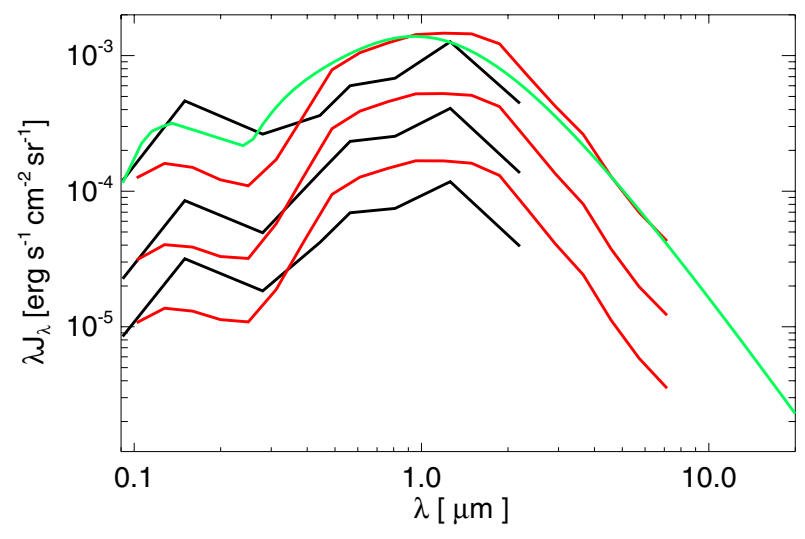

Fig. 4. Spectral energy distribution of the radiation field $\lambda J_{\lambda}$ for $R=$ $11 \mathrm{kpc}$ and for $z=0,8,22 \mathrm{kpc}$ (from top to bottom). Red lines represent the radiation field obtained with the TRADING model, black lines represent the model described in this paper and the green line represents the galactic radiation field in the solar vicinity obtained by Mathis et al. (1983) rescaled to have the same $G_{0}$ that we obtain in our model at $(R[\mathrm{kpc}], z[\mathrm{kpc}])=(11,0)$.

between the two models for the radiation field at $R=3 h_{\mathrm{s}}$. This is due to the fact that in the TRADING model there is a cutoff in the stellar distribution at about $R=13 \mathrm{kpc}$ which is necessary to fit the dust SED with the observations while in the model described in this paper we set a cutoff at $3 h_{\mathrm{d}} \sim 24 \mathrm{kpc}$.

Figure 4 shows the spectral distribution of the radiation field $\lambda J_{\lambda}$ (red lines: TRADING model, black lines: our model and green line: Mathis et al. 1983) for a radial distance $R=$ $2 h_{\mathrm{s}} \approx 11 \mathrm{kpc}$ and for vertical distances $z=0,8,22 \mathrm{kpc}$ (from top to bottom). In order to compare the radiation field that we calculate in this paper with the radiation field in the solar vicinity we use the parameter $G_{0}$ :

$G_{0}=\frac{\int_{91.2 \mathrm{~nm}}^{207 \mathrm{~nm}} J_{\lambda} \mathrm{d} \lambda}{\int_{91.2 \mathrm{~nm}}^{207 \mathrm{~nm}} J_{\text {Mathis }, \lambda} \mathrm{d} \lambda}$

The value of $G_{0}$ at $(R[\mathrm{kpc}], z[\mathrm{kpc}])=(11,0)$ for our model is $G_{0}=1.47$. The green line in Fig. 4 refers to the radiation field in the solar vicinity but rescaled imposing the same $G_{0}$ calculated above. We notice that the shape of the radiation field in the solar vicinity is similar to the radiation field we calculate for NGC 891. In our model we compute the radiation field only up to the $K$ band, while in the solar vicinity the radiation field is known up to $10^{5} \mu \mathrm{m}$. This does not affect the resulting SED since most of the emitted power is in the range $0.091-0.25 \mu \mathrm{m}$.

The plot in Fig. 5 shows the value of $G_{0}$ as a function of the distance from the galactic plane at a radial distance $R=0 \mathrm{kpc}$ (dotted line) and $R=22 \mathrm{kpc}$ (solid line) as calculated by our model.

\section{PAH dissociation probability}

As a consequence of the absorption of a photon or a collision with an electron, the internal energy of a PAH increases and this leads to the excitation of the entire molecule. The energy transferred to the molecule is spread over the vibrational modes and can lead to the dissociation of the molecule or to radiative relaxation.

In this study we are interested in the emission, for a given dust distribution, in different environments and for now we neglect time dependent effects. To do this, we estimate the probability that a PAH will dissociate before it can emit a sufficient 


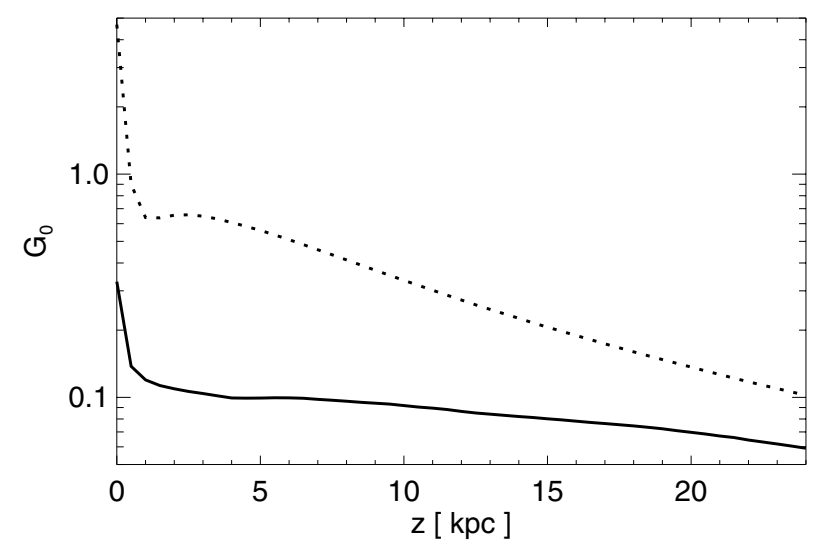

Fig. 5. $G_{0}$ as a function of the distance from the galactic plane for a radial distance $R=0 \mathrm{kpc}$ (dotted line) and $R=22 \mathrm{kpc}$ (solid line) as calculated by our model.

amount of the absorbed energy as IR photons (dissociation probability). If this probability in a given environment is high then destruction dominates over radiative relaxation and we are unlikely to detect the emission from a PAH of this size in this environment. The dissociation probability varies with the size of the PAH. For large molecules the transferred energy can be spread over a larger number of vibrational modes and therefore the dissociation probability for a big molecule is lower than that for a smaller molecule. We set the probability threshold at 0.01 . A molecule with a dissociation probability equal to the probability threshold will be the smallest molecule considered in the size distribution, molecules smaller than this are assumed to be quickly destroyed and unable to emit enough to be detected.

We use the approach developed by Micelotta et al. (2010). What is important in this approach is that, for the grain that we consider, the time between two collisions is longer than the time needed to cool down. We verify this assumption in Sect. 8. We treat the problem in the microcanonical ensemble, the transferred energy, $E_{\mathrm{tr}}$, is related to the effective internal energy of the system, $E_{\mathrm{eff}}$, by:

$E_{\mathrm{eff}} \simeq 0.17\left(\frac{E_{\mathrm{tr}}(\mathrm{eV})}{N_{\mathrm{C}}}\right)^{0.4}\left(1-0.2 \frac{E_{0}(\mathrm{eV})}{E_{\mathrm{tr}}(\mathrm{eV})}\right) \mathrm{eV}$,

where $E_{0}$ is the binding energy of the fragment, which we set equal to $4.6 \mathrm{eV}$ as a standard value (Micelotta et al. 2010). Then the unimolecular dissociation rate, $k_{\text {diss }}$, written in Arrhenius form is given by:

$k_{\text {diss }}=k_{0}\left(E_{\mathrm{eff}}\right) \exp \left[-E_{0} / E_{\mathrm{eff}}\right]$,

where $k_{0}$ has a weak dependency on the internal energy and in this calculation is considered constant and equal to $1.4 \times$ $10^{16} \mathrm{~s}^{-1}$. As per Micelotta et al. (2010), we consider that each emitted photon has an energy $\Delta \epsilon=0.16 \mathrm{eV}$ and that the maximum number of photons emitted scales with the number of carbon atoms, $N_{\mathrm{C}}$, in the PAH as $n_{\max }=N_{\mathrm{C}} / 5$. This leads to Eq. (17) in Micelotta et al. (2010):

$P\left(n_{\max }\right)=\left(n_{\max }+1\right) \frac{k_{0} \exp \left(-E_{0} / E_{\mathrm{av}}\right)}{k_{\mathrm{IR}}}$,

where $P\left(n_{\max }\right)$ is the total un-normalized dissociation probability after the ejection of $n_{\max }$ photons, the average temperature, $E_{\mathrm{av}}$, is chosen as the geometric mean $\left(E_{\mathrm{av}}=\sqrt{E_{\mathrm{tr}} \times E_{n_{\max }}}\right)$, $E_{n_{\max }}=\left(E_{\mathrm{tr}}-n_{\max } \times \Delta \epsilon\right)$ and $k_{\mathrm{IR}}$ is assumed to be 100 photons s$^{-1}$
(Jochims et al. 1994). We apply this method to put a lower limit on the dust population sizes for both photon and electron collisional heating.

Considering only photon heating in the solar vicinity, the transferred energy, $E_{\text {tr }}$ is the energy of the single absorbed photon. For a given $E_{\mathrm{tr}}$ we can calculate the corresponding $P\left(n_{\max }\right)$. We then need to average $P\left(n_{\max }\right)$ over the interstellar radiation field (ISRF) distribution as follows:

$\overline{P\left(n_{\max }\right)}=\frac{\int_{0}^{13.6 \mathrm{eV}} P\left(n_{\max }\right)_{E_{\mathrm{tr}}} J_{\mathrm{ISRF}}(E) \mathrm{d} E}{\int_{0}^{13.6 \mathrm{eV}} J_{\mathrm{ISRF}}(E) \mathrm{d} E}$.

This leads to a probability lower than 0.01 only for PAHs with $N_{\mathrm{C}} \gtrsim 30$, which is in accordance with previous estimates (Jochims et al. 1994).

We now consider electron collisional heating. In this case $E_{\mathrm{tr}}=\zeta(E) E$ and we need to average $P\left(n_{\max }\right)$ over the MaxwellBoltzmann distribution, $f_{\mathrm{M}}(E)$ :

$\overline{P\left(n_{\max }\right)}=\int_{0}^{\infty} P\left(n_{\max }\right)_{E_{\mathrm{tr}}} f_{\mathrm{M}}(E) \mathrm{d} E$.

For example, if we take a gas temperature $T_{\text {gas }}=10^{6}-10^{7} \mathrm{~K}$, the transferred energy is much higher than in the case of photon absorption and this method leads to a size limit of $N_{\mathrm{C}} \approx 200$. This size limitation can be applied to both PAHs and hydrogenated amorphous carbon, a-C $(: \mathrm{H})$, grains since the structure of the small a-C $(: \mathrm{H})$ grains is very close to that of the PAHs, i.e. they are both highly aromatic (Jones 2012). This number of carbon atoms corresponds to a PAH of radius $a \approx 0.77 \mathrm{~nm}$ and an a-C:H grain of radius $a \approx 0.82 \mathrm{~nm}$ with the density and the geometry (both PAHs and a-C:H grains considered as spherical) used in the Compiègne et al. (2011) dust model. As shown by Bocchio et al. (2012) the 2D and 3D grain approaches are equivalent up to radii as large as $\approx 5 \mathrm{~nm}$.

\section{Grain charge effects}

The effect of grain charging in such a hot and tenuous medium has been studied by Draine \& Salpeter (1979). For temperatures $T_{\text {gas }} \sim 10^{6}-10^{7} \mathrm{~K}$ the charging due to the emission of secondary electrons is more important than the charging due to photoelectric effect and can lead very quickly to high electric potentials (e.g., $\sim 30 \mathrm{~V})$. In the case of small grains, they will be quickly destroyed by the field ion emission process. On the other hand, the Coulomb focusing effect is not important at these high temperatures because the electrostatic energy of the grain is always much smaller than the gas thermal energy.

We give an estimate of the timescale needed for a small grain $(a=1 \mathrm{~nm})$ to reach a potential of $30 \mathrm{~V}$ in a gas at $T_{\text {gas }} \sim 10^{7} \mathrm{~K}$ and $n_{\mathrm{H}}=10^{-3} \mathrm{~cm}^{-3}$. The timescale between two collisions with an electron can be expressed as:

$\tau_{\text {coll }}=\frac{1}{n_{\mathrm{H}} \sigma \mathrm{v}_{\mathrm{e}}}$

where $\sigma=\pi a^{2}=\pi \times 10^{-14} \mathrm{~cm}^{2}$, and $v_{\mathrm{e}}=\sqrt{2 k_{\mathrm{B}} T_{\text {gas }} / m_{\mathrm{e}}} \approx$ $1.9 \times 10^{9} \mathrm{~cm} / \mathrm{s}$. This leads to a timescale $\tau_{\text {coll }} \approx 0.6 \mathrm{yr}$. To obtain the timescale for the grain to reach the potential of $30 \mathrm{~V}$ we need to multiply our result by the yield ( $Y_{\mathrm{sec}}$ following Draine \& Salpeter 1979 is approximately unity) and by the number of electrons that we need to extract $\left(\sim 70 \mathrm{e}^{-}\right)$. Therefore the time needed for a small grain to be torn apart by field ion emission is 


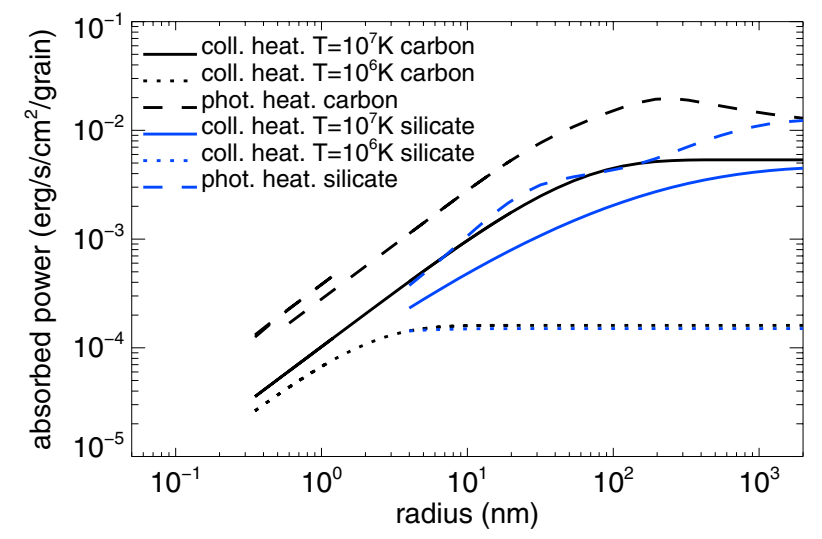

Fig. 6. Power absorbed per unit area per grain due to photon and collisional heating at $z=0$ and $R=14 \mathrm{kpc}$ in NGC 891, where, according to our model, $G_{0}=1$. The collisional heating has been computed as if the plane of the galaxy was consisted of a hot gas with $T=10^{6}$ and $10^{7} \mathrm{~K}$ and for $n_{\mathrm{H}}=10^{-3} \mathrm{~cm}^{-3}$. In the three black lines there is a discontinuity around $1 \mathrm{~nm}$ because of the transition between PAHs and a-C:H grains.

about $\tau_{\text {fie }} \approx 40$ yr. Given that this timescale is so short we can consider these grains to be instantaneously destroyed.

Draine \& Salpeter (1979) calculated the minimum grain radius that will not be destroyed by the high electric potential. For $T_{\text {gas }}=10^{7} \mathrm{~K}, n_{\mathrm{H}}=10^{-3} \mathrm{~cm}^{-3}$ and assuming a maximum effective tensile stress of $7 \times 10^{10} \mathrm{dyne}^{-2}$, the minimum grain radius allowed is $a_{\min } \approx 2 \mathrm{~nm}$.

The effect of grain charge in such a high temperature gas is therefore critical for small grains and will therefore be explored in detail in a future study.

The effect of charging due to the absorption of the X-ray photons produced in such a hot and tenuous medium was taken into account by Draine \& Salpeter (1979) and was considered to be completely negligible with respect to the effect of charging due to secondary electron emission.

\section{Photon vs. electron collisional heating}

In this work we use the Compiègne et al. (2011) dust model where all of the molecules and grains are considered to be spherical. We compare the two dust heating processes: collisions with electrons and the absorption of photons. In Fig. 6 we show the absorbed energy per unit area per grain due to photon and collisional heating as a function of the grain/molecule radius. This plot refers to the point $(R[\mathrm{kpc}], z[\mathrm{kpc}])=(14,0)$ in the galaxy NGC 891 where, according to our model, $G_{0}=1$. The electron collisional heating has been calculated as if the plane of the galaxy contains only a hot gas with $T=10^{6}-10^{7} \mathrm{~K}$ and $n_{\mathrm{H}}=$ $10^{-3} \mathrm{~cm}^{-3}$. The discontinuity in the black lines around $a \sim 1 \mathrm{~nm}$ is due to the transition between PAHs and a-C:H grains.

We notice that in the case of a gas temperature of $10^{6} \mathrm{~K}$ the electron collisional heating for carbonaceous and silicate grains are very close to one other. This is because the peak of the Maxwell-Boltzmann distribution, corresponding to $T_{\mathrm{gas}}=$ $10^{6} \mathrm{~K}$, is in the linear portion of the transferred energy function for both silicates and carbonaceous grains. This is not the case for $T_{\text {gas }}=10^{7} \mathrm{~K}$, where the electron energy can be higher than the threshold of the transferred energy function. Furthermore, in the latter case, the electron collisional heating is less than an order of magnitude lower than the photon heating for each of the sizes.

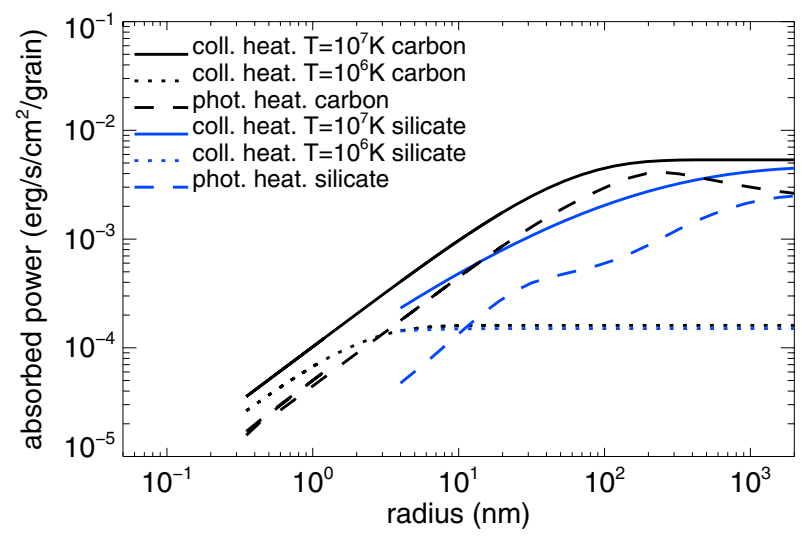

Fig. 7. Power absorbed per unit area per grain due to photon and collisional heating at $z=3.5 \mathrm{kpc}$ and $R=22 \mathrm{kpc}$ in NGC 891, where, according to our model, $G_{0}=0.1$. The collisional heating has been computed for IGM gas with a temperature $T_{\text {gas }}=10^{6}$ and $10^{7} \mathrm{~K}$ and a proton density $n_{\mathrm{H}}=10^{-3} \mathrm{~cm}^{-3}$. In the three black lines there is a discontinuity around $1 \mathrm{~nm}$ because of the transition between PAHs and a-C:H grains.

From Fig. 5 we have that $G_{0}$ is about 0.1 at $(R[\mathrm{kpc}]$, $z[\mathrm{kpc}])=(22,3.5)$. Therefore photon heating should be an order of magnitude lower than in the previous case and should be comparable with the collisional heating. Figure 7 represents the power absorbed per unit area per grain due to both photon absorption and collisional heating in the galaxy NGC 891 at $(R$ $[\mathrm{kpc}], z[\mathrm{kpc}])=(22,3.5)$ where $G_{0} \approx 0.1$. We notice that, in this region, the collisional heating is larger than the photon heating and therefore must be taken into account. Thus, at relatively large vertical distances above the plane of a galaxy, electron collisional heating could be as important, or more important than, photon heating. However, far from a galaxy, in the IGM, it is likely that dust may not survive long (e.g. Bocchio et al. 2012) and because of this we are currently evaluating the gas and dust dynamics during the ram pressure stripping.

\section{DustEM code}

The DustEM code is a numerical tool presented by Compiègne et al. (2011), which calculates the dust SED for a given dust model and given ISRF. We have now included in DustEM the heating of dust due to collisions with electrons (see Appendix A for details). The corresponding power (Eq. (1)) is added to the photon heating power and the grain temperature distribution is derived using the method described by Désert et al. (1986).

Even if DustEM calculates the grain temperature distribution for each grain size, it is important to know if a grain of a given size in a certain environment is stochastically heated or is in thermal equilibrium. For example, to estimate the PAH dissociation probability in Sect. 5, we implicitly assumed that PAHs are not in thermal equilibrium but that they experience significant temperature fluctuations. First of all, we need to calculate the average time between the absorption of two photons, $\tau_{\mathrm{abs}}$, and the average time between two collisions with electrons, $\tau_{\text {coll }}$. Then we need to compare these average times with the cooling time, $\tau_{\text {cool }}$. If the cooling time is larger than the time between collisions or photon absorptions, then the grain will tend to a thermal equilibrium, otherwise it will be stochastically heated.

The average time between the absorption of two photons is given by:

$\tau_{\text {abs }}^{-1}=\pi a^{2} \int_{0}^{\infty} \frac{4 \pi J_{\lambda} Q_{\lambda} \lambda}{h c} \mathrm{~d} \lambda$ 
where $\pi a^{2} Q_{\lambda}$ is the absorption cross section for a given wavelength $\lambda$. The average time between collisions with electrons is given by:

$\tau_{\text {coll }}^{-1}=\pi a^{2} \sqrt{\frac{3 k_{\mathrm{B}} T_{\mathrm{gas}}}{m_{\mathrm{e}}}} n_{\mathrm{H}}$

where $m_{\mathrm{e}}$ is the electron mass.

As a consequence of the absorption of the most energetic photons or the most energetic collisions with the electrons ${ }^{2}$, the internal energy of a grain will increase and its temperature will reach the highest value $T_{\max }$. We define the cooling time as the time needed to cool down from the temperature $T_{\max }$ to a temperature where the radiative emission is negligible with respect to the radiative emission at the highest temperature. We calculate the highest temperature reached by a grain as a consequence of the injection (due to photon absorption of collisional heating) of an energy $E_{\text {in }}$ by:

$E_{\text {in }}=\int_{0}^{T_{\max }} C\left(T_{\mathrm{d}}\right) \mathrm{d} T_{\mathrm{d}}$

As given by Dwek (1986) we can write that, between two heating events, a grain cools at a rate:

$$
\begin{aligned}
\mathrm{d} T_{\mathrm{d}} / \mathrm{d} t & =L_{\mathrm{d}} / C\left(T_{\mathrm{d}}\right) \\
& =4 \pi a^{2}\left\langle Q_{\mathrm{abs}}\right\rangle_{T_{\mathrm{d}}} \sigma T_{\mathrm{d}}^{4} / C\left(T_{\mathrm{d}}\right)
\end{aligned}
$$

where $L_{\mathrm{d}}$ is the the rate at which a dust particle radiates:

$$
\begin{aligned}
L_{\mathrm{d}} & =4 \pi a^{2} \int_{0}^{\infty} \pi B_{\lambda}\left(T_{\mathrm{d}}\right) Q_{\lambda}(a) \mathrm{d} \lambda \\
& =4 \pi a^{2} \sigma T_{\mathrm{d}}^{4}\left\langle Q_{\mathrm{abs}}\right\rangle_{T_{\mathrm{d}}}
\end{aligned}
$$

with $B_{\lambda}$ the Planck function, $\sigma$ the Stefan-Boltzmann constant and $\left\langle Q_{\mathrm{abs}}\right\rangle_{T_{\mathrm{d}}}$ the Planck-averaged value of $Q_{\lambda}(a)$. Following this approach, the cooling time can be written as:

$$
\begin{aligned}
\tau_{0} & =\int_{T_{\mathrm{i}}}^{T_{\max }} \mathrm{d} T_{\mathrm{d}} /\left|\left(\mathrm{d} T_{\mathrm{d}} / \mathrm{d} t\right)\right| \\
& =\int_{T_{\mathrm{i}}}^{T_{\max }} \frac{C\left(T_{\mathrm{d}}\right) \mathrm{d} T}{L_{\mathrm{d}}}
\end{aligned}
$$

where $T_{\mathrm{i}}$ is the initial temperature of the grain $\left(T_{\mathrm{i}}=0.1 \mathrm{~K}\right.$ in DustEM code). We note that this definition of the cooling time depends on the value of the minimum temperature of the grain and is dominated by the time spent close to the lowest temperatures. We therefore define the cooling time in this way:

$$
\begin{aligned}
\tau_{\text {cool }}=\int_{\ln T_{\mathrm{i}}}^{\ln T_{\max }} \frac{C\left(T_{\mathrm{d}}\right) T_{\mathrm{d}} L_{\mathrm{d}} \frac{\mathrm{d} P}{\mathrm{~d} \ln T_{\mathrm{d}}} \mathrm{d} \ln T_{\mathrm{d}}}{L_{\mathrm{d}} \int_{\ln T_{\mathrm{i}}}^{\ln T_{\max }} L_{\mathrm{d}} \frac{\mathrm{d} P}{\mathrm{~d} \ln T_{\mathrm{d}}} \mathrm{d} \ln T_{\mathrm{d}}} \\
=\frac{\int_{\ln T_{\mathrm{i}}}^{\ln T_{\max }} C\left(T_{\mathrm{d}}\right) T_{\mathrm{d}} \frac{\mathrm{d} P}{\mathrm{~d} \ln T_{\mathrm{d}}} \mathrm{d} \ln T_{\mathrm{d}}}{\int_{\ln T_{\mathrm{i}}}^{\ln T_{\max }} L_{\mathrm{d}} \frac{\mathrm{d} P}{\mathrm{~d} \ln T_{\mathrm{d}}} \mathrm{d} \ln T_{\mathrm{d}}},
\end{aligned}
$$

where $\frac{\mathrm{d} P}{\mathrm{~d} \ln T_{\mathrm{d}}}$ is the probability for a grain to be at a certain temperature. In this way, $\tau_{\text {cool }}$ is not sensitive to the minimum temperature that we consider and represents the time needed for a grain to cool to a temperature where the radiative emission is

\footnotetext{
2 The most energetic collisions with the electrons correspond, for a given size, to the maximum deposited energy i.e. the maximum of the transferred energy function $E \zeta(E)$.
}

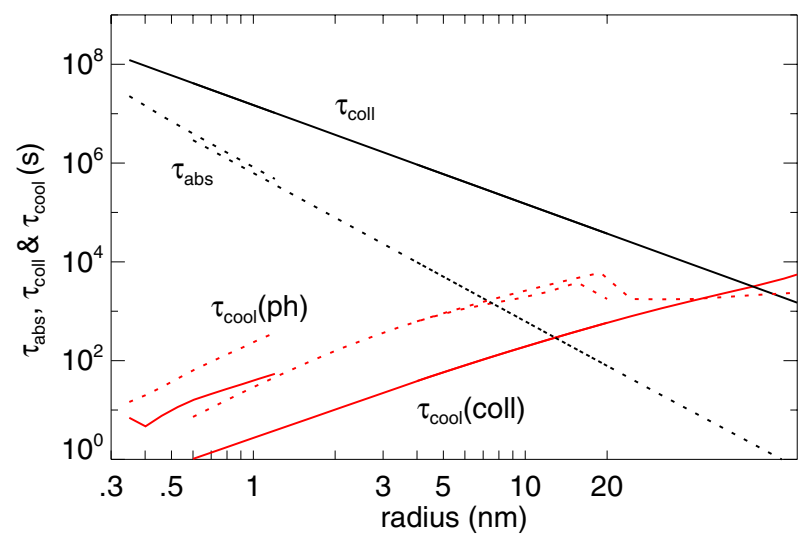

Fig. 8. Photon absorption time $\left(\tau_{\text {abs }}\right)$, electron collision time $\left(\tau_{\text {coll }}\right)$ and cooling times $\left(\tau_{\text {cool }}(\right.$ coll $)$ and $\left.\tau_{\text {cool }}(\mathrm{ph})\right)$ for carbonaceous grains with radius from 0.3 to $30 \mathrm{~nm}$. In the case of photon absorption, we assume a radiation field with $G_{0}=1$, while in the case of electron collisions, $T_{\text {gas }}=10^{7} \mathrm{~K}$ and $n_{\mathrm{H}}=10^{-3} \mathrm{~cm}^{-3}$ have been assumed. $\tau_{\text {cool }}$ (coll) represents the cooling time in the case of collisional heating only whilst $\tau_{\text {cool }}(\mathrm{ph})$ represents the cooling time in the case of photon absorption only. In all the three lines there is a discontinuity between $0.6 \mathrm{~nm}$ and $1.2 \mathrm{~nm}$ because of the transition between PAHs and a-C:H grains.

negligible with respect to the radiative emission at the maximum temperature. If we consider the electron collisional heating only we call the cooling time $\tau_{\text {cool }}($ coll $)$, while if only the photon heating is present we call the cooling time $\tau_{\text {cool }}(\mathrm{ph})$.

In Fig. 8 we show $\tau_{\text {coll }}, \tau_{\text {abs }}, \tau_{\text {cool }}($ coll $)$ and $\tau_{\text {cool }}(\mathrm{ph})$. There is a discontinuity in the cooling time at a size of about $a \approx 1 \mathrm{~nm}$ because of the transition between the PAHs and a-C:H grains. The other two small discontinuities at $a \approx 4 \mathrm{~nm}$ and $a \approx 20 \mathrm{~nm}$ are due to small differences in the adopted size distributions (power law and $\log$ normal) of a-C:H grains. We notice that for a grain of about $a_{1} \approx 7 \mathrm{~nm}$, the cooling time, in the case of photon heating only, equals the absorption time. This means that, if we assume no collisional heating, grains with radii smaller than $a_{1}$ are stochastically heated, while larger grains tend to be in thermal equilibrium. If we consider the electron collisional heating only, the intersection between the two curves corresponds to a grain radius $a_{2} \approx 27 \mathrm{~nm}$. When both processes occur, the average cooling time is very similar to the cooling time in the case of photon heating only, because the collision with an electron, for each of the grain sizes, is much rarer than the absorption of a photon. The average time between two events (be they electron collisions or photon absorptions) can be calculated as follow (if they are independent, which is the case here):

$\tau_{\text {event }}=\left(\frac{1}{\tau_{\mathrm{abs}}}+\frac{1}{\tau_{\text {coll }}}\right)^{-1}$.

Since electron collisions are much rarer than photon absorptions $\tau_{\text {event }} \approx \tau_{\text {abs }}$ and therefore the intersection between the two lines corresponding to the cooling time, and average time between two events, would be at around $a_{\text {both }} \approx 7 \mathrm{~nm}$. In Sect. 5 we made the assumption that a-C:H grains smaller than $N_{\mathrm{C}} \approx 200$ (corresponding to $a_{\mathrm{a}-\mathrm{C}: \mathrm{H}}=0.77 \mathrm{~nm}$ ) were stochastically heated. We therefore confirm that this assumption is valid.

Using DustEM we obtain the dust temperature distribution for each grain size. We consider a small PAH $(a=0.77 \mathrm{~nm})$ and we compare the temperature distribution in the case of photon heating, collisional heating or both processes. As we can see in Fig. 8 such a grain is stochastically heated in all cases. This is confirmed by the shape of the temperature distributions 


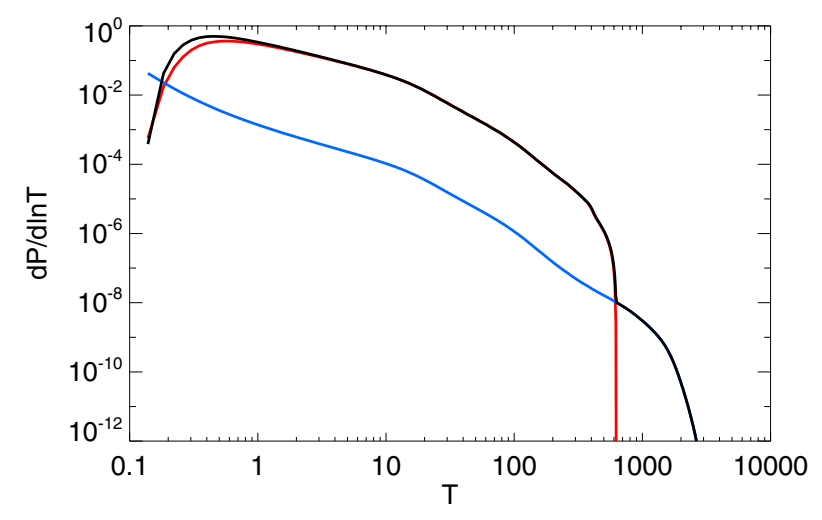

Fig. 9. $\mathrm{d} P / \mathrm{d} \ln T$ (probability for a grain to be at a certain temperature) in the case of a stochastically-heated PAH molecule (radius $a=0.765 \mathrm{~nm}$ ) assuming a gas temperature $T_{\text {gas }}=10^{7} \mathrm{~K}$, a gas proton density $n_{\mathrm{H}}=$ $10^{-3} \mathrm{~cm}^{-3}$ and $G_{0}=1$. We notice that the probability distribution in the case of dust heating due to both collisions and photon absorption (black line) is, for most of the temperatures, the sum of the probability distribution due to only photon heating (red line) and the probability distribution due to collisional heating (blue line).

in Fig. 9, which shows the probability for a grain to be at a certain temperature $(\mathrm{d} P / \mathrm{d} \ln T)$ with respect to the range of temperatures that the grains can assume. We assume a gas temperature $T_{\text {gas }}=10^{7} \mathrm{~K}$, a gas proton density $n_{\mathrm{H}}=10^{-3} \mathrm{~cm}^{-3}$ and $G_{0}=1$. We notice that, for most temperatures, we have:

$\mathrm{d} P / \mathrm{d} \ln T$ (collision + photon $)=\mathrm{d} P / \mathrm{d} \ln T$ (collision $)+\mathrm{d} P / \mathrm{d} \ln T$ (photon).

If we consider a grain that is large enough to be in thermal equilibrium with collisional heating only, then in this case we can analytically calculate the equilibrium temperature, equating the rate of absorbed energy $H$ (see Eq. (1)), to the rate at which a dust particle radiates, $L_{\mathrm{d}}$ (see Eq. (23)). If we assume $Q_{\lambda} \sim \lambda^{-\beta}$, where $\beta$ is the opacity power law index (in our case $\beta \approx 1.55$, see Compiègne et al. 2011), the predicted equilibrium temperature is:

$T_{\mathrm{d}} \sim\left(\frac{n_{\mathrm{H}}\langle v(E) \zeta(E) E\rangle}{4 \sigma}\right)^{\left(\frac{1}{4+\beta}\right)}$

where the averaging is over the Maxwell-Boltzmann distribution. We consider for example a grain of radius $a=0.32 \mu \mathrm{m}$ and look at the equilibrium temperature for $n_{\mathrm{H}}=10^{-4}-10^{-1} \mathrm{~cm}^{-3}$. We can fit the obtained values with a power law and obtain:

$T_{\text {equi }} \propto n_{\mathrm{H}}^{0.184}$

In Fig. 10 we show the equilibrium temperature with respect to the proton density of the gas. The black plus signs represent the values obtained with DustEM (with the method described by Désert et al. 1986) while the red line is the fit to these values given by Eq. (28). The value of the exponent, $\gamma_{\text {DustEM }}=0.184$, is close to what we would expect analytically, $\gamma_{\text {th }}=0.180$, they differ by only $2 \%$. This small difference can be explained by the fact that DustEM considers a temperature distribution rather than single equilibrium temperature and the parameter $\beta$ is not exactly 1.55 as assumed in the analytical approximation.

\section{Results}

We apply the present model to the region $(R[\mathrm{kpc}], z[\mathrm{kpc}])=$ $(14,0)$ in NGC 891 where, following the method described above, $G_{0}=1$. The plot in Fig. 11 shows the dust SED calculated with DustEM due to only photon heating without (red line)

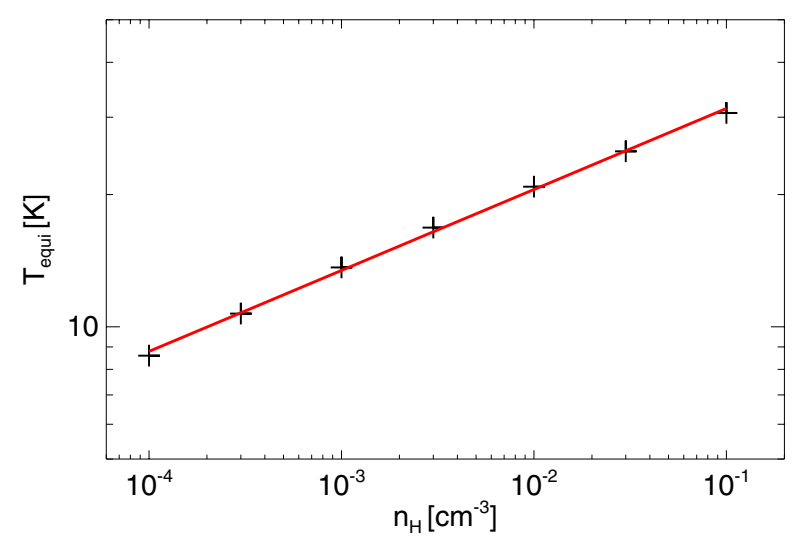

Fig. 10. Equilibrium temperature, $T_{\text {equi }}$, with respect to the proton density, $n_{\mathrm{H}}$, for a gas temperature $T_{\text {gas }}=10^{7} \mathrm{~K}$ and for a grain of radius $a=0.32 \mu \mathrm{m}$, heated only by electron collisions. The black plus signs have been obtained with DustEM and the red line is the straight line fit to these values $\left(T_{\text {equi }} \propto n_{\mathrm{H}}^{0.184}\right)$.

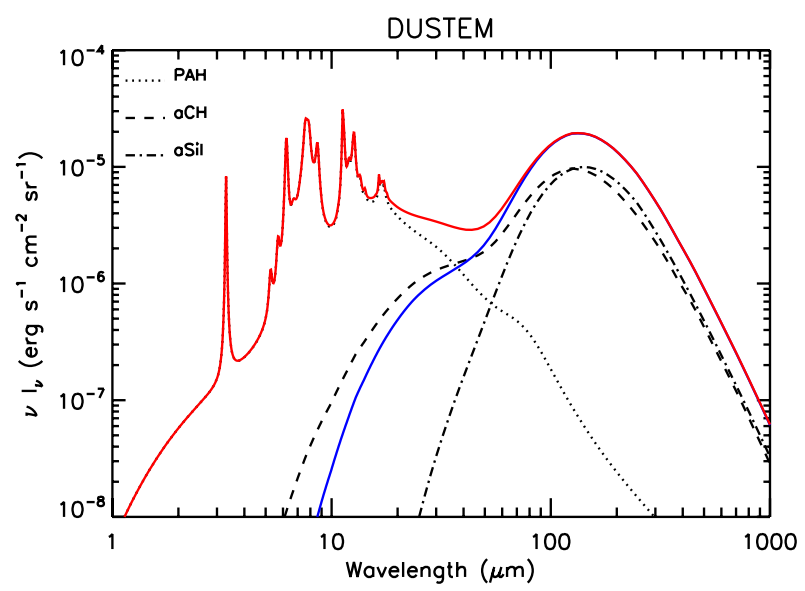

Fig. 11. Dust SED for $G_{0}=1$ in the region $(R[\mathrm{kpc}], z[\mathrm{kpc}])=(14,0)$ in the galaxy NGC 891 for an assumed column density $N_{\mathrm{H}}=10^{20} \mathrm{~cm}^{-2}$. The red and blue lines represent the SED due to photon heating only, with (blue line) and without (red line) the size limitation at $a_{\min }=2 \mathrm{~nm}$ caused by the presence of a gas at $T_{\text {gas }}=10^{7} \mathrm{~K}$ and $n_{\mathrm{H}}=10^{-3} \mathrm{~cm}^{-3}$. Black lines represent the various contribution to the SED shown by the red line.

and with (blue line) the size limitation (in the PAH and a-C:H size distributions) at $a_{\mathrm{min}}=2 \mathrm{~nm}$ (added to show the destructive effect in the case of the presence of a hot gas). The silicate grains considered in the Compiègne et al. (2011) model are big enough $\left(a_{(\min , \text { sil. })}=4 \mathrm{~nm}\right)$ to be unaffected by destruction during heating so no change in the lower limit of the size distribution has been imposed on this population.

The black lines show the different contributions to the SED calculated without any size limitation. The black dashdotted line represents the contribution to the SED coming from silicate grains, the dotted line represents the contribution due to PAH emission, the dashed line represents the contribution from the a-C:H grains while the red line represents the total dust SED (we keep the same style-color coding for all of the SEDs plotted in this paper). Including electron collisional heating in this case would not affect the result, as it is negligible with respect to the photon heating (see Fig. 6). We note that the grain size limitation, imposed as a consequence of grain charging effects leading to destruction, has a major effect on the emission features, in particular since all the PAHs are quickly destroyed, there is no evidence for any PAH features in the dust SED (blue line). 


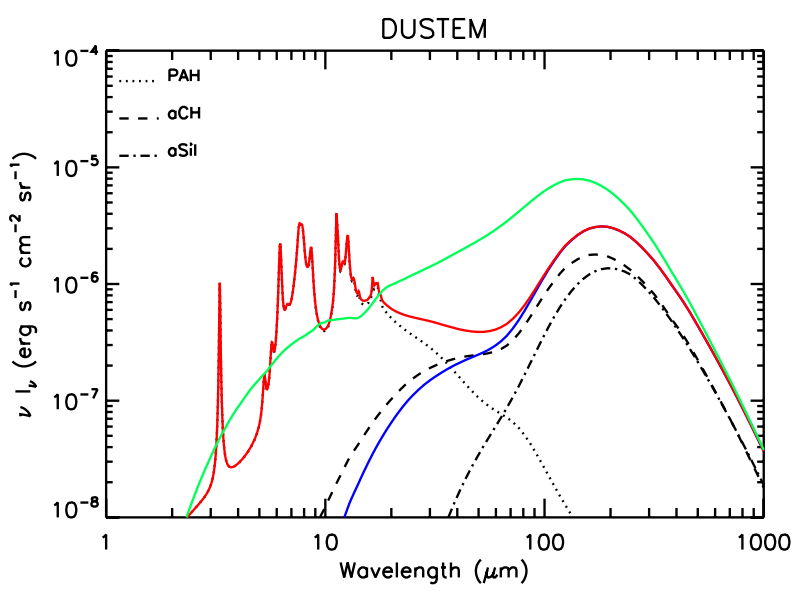

Fig. 12. Dust SED for $G_{0}=0.1$, in the region $(R[\mathrm{kpc}], z[\mathrm{kpc}])=$ $(22,3.5)$ in the galaxy NGC 891 . The red and blue lines represent the SED due to photon heating only, with (blue line) and without (red line) the size limitation at $a_{\min }=2 \mathrm{~nm}$. The green line represents the SED due to both photon and collisional heating with the size limitation at $a_{\text {min }}=2 \mathrm{~nm}$ (we assume the totality of the dust as embedded in the hot gas). Black lines represent the various contribution to the SED shown by the red line. We assume the same column density and gas properties as in Fig. 11.

The plot in Fig. 12 shows the SED in the region $(R[\mathrm{kpc}]$, $z[\mathrm{kpc}])=(22,3.5)$ in the galaxy NGC 891. Following our method, in this region we have a radiation field with $G_{0}=0.1$. The red line shows the SED in the case of photon heating only and with no evolution of the smallest particles. The blue line shows the SED due to only photon heating as in the case of the red line but we here impose a cut in the size distribution at $a_{\min }=2 \mathrm{~nm}$ given by the destructive effect of a hot gas. The green line shows the SED as in the case of the blue line but with the addition of electron collisional heating, as in the case of an IGM gas $\left(T_{\text {gas }}, n_{\mathrm{H}}\right)=\left(10^{7} \mathrm{~K}, 10^{-3} \mathrm{~cm}^{-3}\right)$. The black lines represents the contributions to the red line.

Comparing the green and blue lines in Fig. 12 it is clear that the short wavelength emission $(\lambda<10 \mu \mathrm{m})$ from a hot tenuous gas with low $G_{0}$ can come only from the electron collisional heating of larger grains ( $a>2 \mathrm{~nm}$ ), or equivalently we can say that the ISRF does not have sufficiently high energy photons to heat small grains to high enough temperatures to produce this short wavelength emission. Also, the mid- and far-IR emission are significantly enhanced by the inclusion of electron collisional heating.

Since the value of $a_{\min }$ depends not only on the properties of the gas where dust is embedded, but also on the dynamics of dust in this medium, we plot in Fig. 13 the resulting dust SED (for $G_{0}=0.1, T_{\text {gas }}=10^{7} \mathrm{~K}$ and $n_{\mathrm{H}}=10^{-3} \mathrm{~cm}^{-3}$ ) for different values of $a_{\mathrm{min}}$, ranging from 0.77 to $5 \mathrm{~nm}$. We notice that the dust SED presents PAH features only for $a_{\min }=0.77 \mathrm{~nm}$ because the other values of $a_{\min }$ are larger than that of the PAHs in our model.

\section{Astrophysical implications}

From this work it is clear that the inclusion of electron collisional heating and grain charge effects lead to significant changes in the shape of the IR-mm SED (green and red lines in Fig. 12). The most obvious effect is the complete loss of the aromatic emission bands, in the 3-20 $\mu \mathrm{m}$ wavelength region, due to the destruction of the PAHs. Here this effect is modeled by increasing the minimum grain radius to $2 \mathrm{~nm}$, which is larger than that of PAHs (see Table 1). This result is consistent with the Micelotta et al. (2010) and Bocchio et al. (2012) results but we find that, with

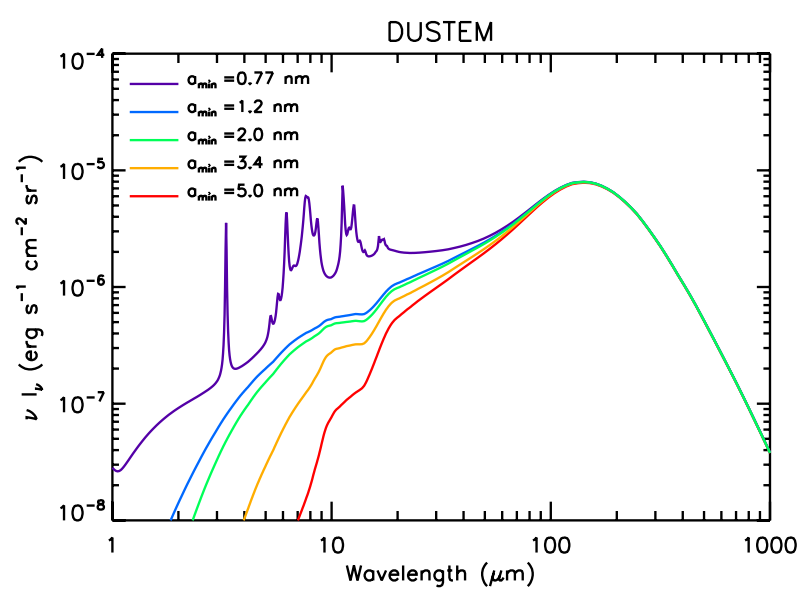

Fig. 13. Dust SED due to both photon $\left(G_{0}=0.1\right)$ and electron collisional heating $\left(n_{\mathrm{H}}=10^{-3} \mathrm{~cm}^{-3}, T_{\text {gas }}=10^{7} \mathrm{~K}\right)$ for different values of $a_{\mathrm{min}}$.

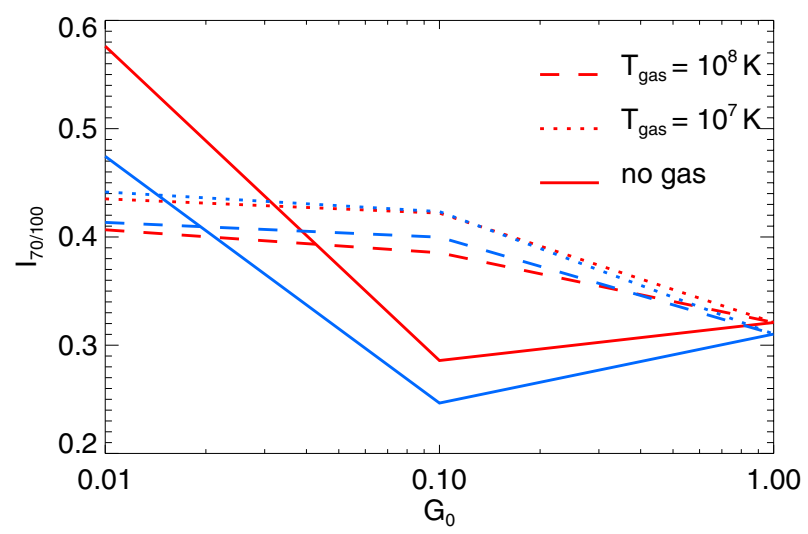

Fig. 14. Ratio $I_{70 / 100}$ computed for the Compiègne et al. (2011) dust model (red lines) and for the Draine \& Li (2007) dust model (blue lines). The gas proton density is kept constant at $n_{\mathrm{H}}=10^{-3} \mathrm{~cm}^{-3}$, the solid lines represent the case of the absence of collisional heating and destruction, while the dotted lines and the dashed lines correspond to a gas temperature of $T_{\text {gas }}=10^{7} \mathrm{~K}$ and $T_{\text {gas }}=10^{8} \mathrm{~K}$ respectively.

the inclusion of grain charge processes, the destructive effect of a hot gas on small particles $(a \sim 1-3 \mathrm{~nm})$ is even more important than in their work.

The next most obvious effect of including electron collisional heating, in a low density and weak radiation field environment, is a shift in the peak wavelength of the big grain emission, from $\sim 200 \mu \mathrm{m}(T \sim 15 \mathrm{~K})$ to $\sim 130 \mu \mathrm{m}(T \sim 23 \mathrm{~K})$, and the appearance of the emission bands at 10 and $18 \mu \mathrm{m}$ due to small, hot silicate grains.

We also note that there is an important change in the slope of the dust SED between $70 \mu \mathrm{m}$ and $100 \mu \mathrm{m}$, which is not affected by small grain size cut-off in our model. We have calculated the PACS $70 \mu \mathrm{m}$ to $100 \mu \mathrm{m}$ band ratio, $I_{70 / 100}$, as a function of $G_{0}$ both with and without electron collisional heating. In Fig. 14 we plot $I_{70 / 100}$ for the Compiègne et al. (2011) dust model (red lines) and the Draine \& Li (2007) dust model (blue lines) as a function of $G_{0}$. The gas proton density is kept constant at $n_{\mathrm{H}}=10^{-3} \mathrm{~cm}^{-3}$, the solid lines represent the case where collisional heating and destruction are ignored (no gas), while the dotted lines and the dashed lines correspond to a gas temperature of $T_{\text {gas }}=10^{7} \mathrm{~K}$ and $T_{\text {gas }}=10^{8} \mathrm{~K}$, respectively. We note that the ratio $I_{70 / 100}$ is significantly affected by the presence of a hot gas for $G_{0}=0.1$. The introduction of dust heating and grain charge destruction effects arising from electron collisions in a hot gas leads to changes 


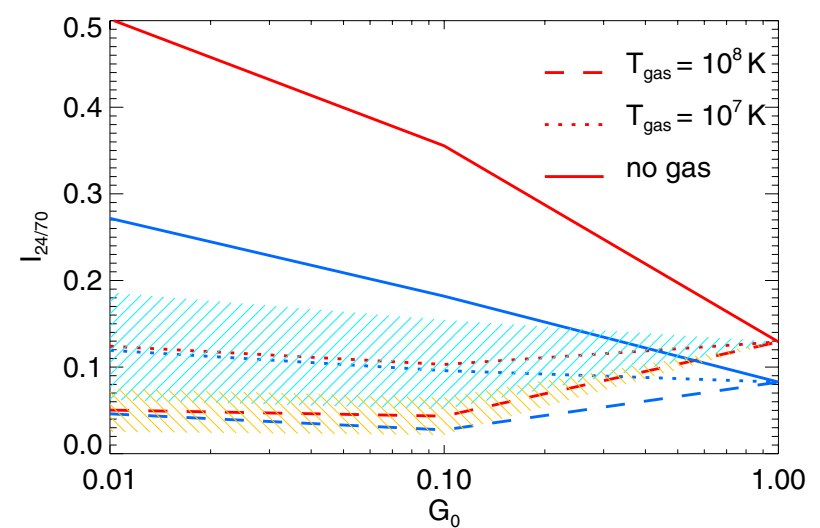

Fig. 15. Same as Fig. 14 but for the ratio $I_{24 / 70}$. The two shaded regions represent the variability in the ratio $I_{24 / 70}$ due to the uncertainty on $a_{\min }$ (see Fig. 13) for our model.

in $I_{70 / 100}$ that could be a good SED diagnostic signature of the presence of a hot gas and a weak radiation field $\left(G_{0} \sim 0.1\right)$. Furthermore we note that the two dust models predict very similar SEDs for wavelengths longer than $10 \mu \mathrm{m}$, i.e. they show the same behaviour (the red and blue lines in Fig. 14), with respect to $G_{0}$, therefore indicating the robustness of our results.

From Figs. 12 and 13 we notice that, regardless the value of $a_{\text {min }}$ we choose in our model, there is a change in the slope between $24 \mu \mathrm{m}$ and $70 \mu \mathrm{m}$. We have therefore calculated the MIPS $24 \mu \mathrm{m}$ to PACS $70 \mu \mathrm{m}$ band ratio $\left(I_{24 / 70}\right)$ for different $G_{0}$ and we plot this in Fig. 15 for the Compiègne et al. (2011) dust model and the Draine \& Li (2007) dust model. We use the same stylecolor code as in Fig. 14. Since in the case of a hot gas electron collisions also destroy the small grains, we therefore calculated the ratio $I_{24 / 70}$ for the different values of $a_{\min }$ shown in Fig. 13. This range of possible values of $a_{\min }$ gives rise to a variability of the ratio $I_{24 / 70}$ of $\pm 50 \%$, which is shown in Fig. 15 by the two shaded regions (light blue for $T_{\text {gas }}=10^{7} \mathrm{~K}$ and orange for $T_{\text {gas }}=10^{8} \mathrm{~K}$ ). Despite the variability of $I_{24 / 70}$ for low $G_{0}$, this band ratio could be, together with $I_{70 / 100}$, a good SED diagnostic signature of the presence of a hot gas acting on dust grains through both electron collisional heating and dust destruction.

It is clear that PAH-sized particles ought to be destroyed in a hot gas through effects arising from extreme charging processes (secondary electron emission, field ion emission and/or Coulomb explosion) on timescales of the order of tens of years (see Sect. 6). Given that this time-scale is so short the aromatic band emission from the outer regions of the entrained clumps will be of extremely limited spatial extent and therefore hard to detect. Therefore any observation of the aromatic emission bands in the SEDs of dust in the IGM local to galaxies would imply very large quantities of dust and gas embedded within entrained dense clumps immersed in the hot gas. These clumps will probably be in pressure equilibrium with the coronal-type IGM gas $\left(n_{\mathrm{H}} T \sim 10^{4} \mathrm{~K} \mathrm{~cm}^{-3}\right)$ if they have densities and temperatures of either the warm inter-cloud medium or the cold neutral medium of the galaxy. However, dynamical processes such as conduction or ablation in the outflowing gas will lead to their eventual evaporation. Any aromatic band emission should therefore come from the shells or halos around embedded clumps, the thickness of which will depend upon the local dynamical conditions and so it is difficult to generalize on the observability of aromatic emission bands in the IGM. Nevertheless, if we consider a dense clump in pressure equilibrium with the hot coronal-type IGM gas $\left(n_{\mathrm{H}} T \sim 10^{4} \mathrm{~K} \mathrm{~cm}^{-3}\right)$ we can estimate

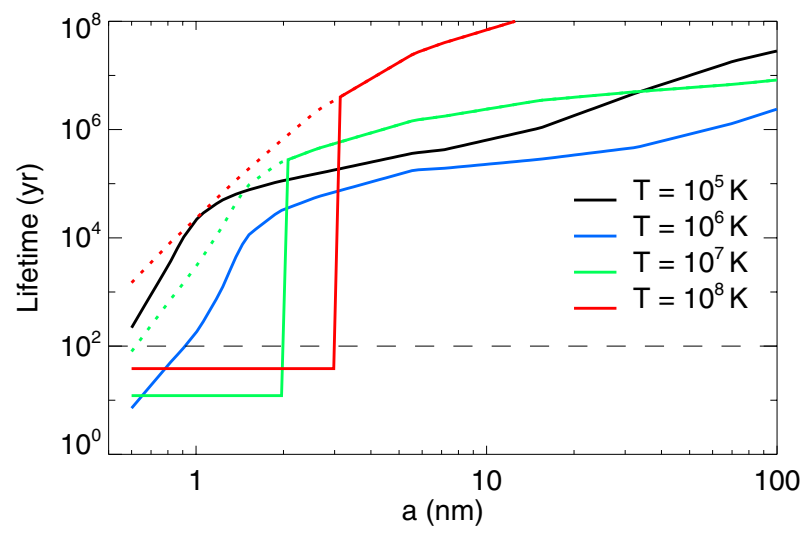

Fig. 16. Lifetime of carbonaceous grains as a function of the grain size for different gas temperatures. The abrupt drop in the lifetime for the red and green curves is due to ion field emission. The dotted lines represent the lifetime of dust without the inclusion of any charge effect. The dashed horizontal line at $10^{2} \mathrm{yr}$ represents the threshold for observability.

the lifetime of small grains for different shell conditions around dense clumps with $T_{\text {clump }}=10^{2} \mathrm{~K}$ and $n_{\mathrm{H}}=10^{2} \mathrm{~cm}^{-3}$. In Fig. 16 we plot the lifetime of grains as a function of size for different gas temperatures. Note that since the shells are assumed to be in pressure equilibrium with the IGM gas, fixing the temperature in a given shell, fixes the proton density as well. In Fig. 16 the the abrupt drop at 2 and $3 \mathrm{~nm}$, for temperatures of $10^{7}$ and $10^{8} \mathrm{~K}$ respectively, is due to the effect of Coulomb explosion/ion field emission, which play an important role in dust destruction leading to even shorter lifetimes than those predicted by Micelotta et al. (2010) and Bocchio et al. (2012).

We find that emission from grains smaller than $2-3 \mathrm{~nm}$ will be difficult to detect in the IGM near and far from galaxies because the small particle destruction time is rather short. On the other hand, if we assume that in order to be observable a grain must have a lifetime $>10^{2} \mathrm{yr}$, then, in the lower density evaporating regions around entrained clumps, emission from grains larger than $2-3 \mathrm{~nm}$ ought to be observable in a gas with $n_{\mathrm{H}} T \sim 10^{4} \mathrm{~K} \mathrm{~cm}^{-3}$. We note that shells with $T_{\text {gas }}<10^{5} \mathrm{~K}$ the dust lifetime is $\gg 10^{8} \mathrm{yr}$. Furthermore, if the evaporation dynamics of these dense clumps is fast, as we clearly see from Fig. 16, then we could observe dust emission from larger grains $(a \gtrsim 3 \mathrm{~nm})$ even in a hot and diffuse gas at $T_{\text {gas }}=10^{8} \mathrm{~K}$ and $n_{\mathrm{H}}=10^{-4} \mathrm{~cm}^{-3}$.

In conclusion, a consideration of the grain charging processes acting on dust embedded within a hot gas, whether in the hot gas itself or ablated from entrained, dense clumps, turns out to be absolutely critical in determining their destruction timescale and hence their observability.

\section{Conclusions}

In hot coronal type gas, for high gas temperatures $T_{\text {gas }}=$ $10^{7}-10^{8} \mathrm{~K}$, the collisions between dust and fast electrons can lead to the heating, destruction and charging of grains. As we have shown, these three processes dramatically affect the dust SED.

In the case of a hot and tenuous gas with $\left(n_{\mathrm{H}}, T_{\text {gas }}\right)=$ $\left(10^{-3} \mathrm{~cm}^{-3}, 10^{7} \mathrm{~K}\right)$, the power absorbed per grain is comparable to the power absorbed per grain due to photon heating in a region where $G_{0} \approx 0.1$. In this environment, we calculate that the charging due to emission of secondary electrons is important and leads to high electric potentials, therefore destroying the smallest 
grains ( $a<2 \mathrm{~nm}$ ) very quickly. We set the size distribution lower limit to $a=2 \mathrm{~nm}$ but we do explore the changes in the SED due to the uncertainty of this parameter. This change in the size distribution, together with the extra energy input due to electron collisions, leads to important differences in the mid- and far-IR part of the SED. In particular, including electron collisional heating leads to an enhancement in the ratio $I_{70 / 100}$ with respect to the case of photon heating only in weak radiation fields (with $\left.G_{0}=0.1\right)$.

In our model we treated the problem in a stationary way: in the case of the presence of a hot gas, we derive a lower limit to the size distribution, which is determined by the radius where the grains are too small to survive long enough to be detected. However, a full analysis really needs to take into account the time dependency of the dust population evolution, which can be calculated at each instant using the destruction rates given by Bocchio et al. (2012) (with charge effects included), and the relevant dust dynamics.

In the case of ram pressure stripped galaxies, our results for the electron collisional heating and collisional destruction represent an upper limit because we assumed that dust was embedded in the IGM gas. In reality the dust could be embedded in a gas which is a mix of IGM gas and HI gas ejected from the stripped galaxy. Therefore a detailed analysis of the dynamics of dust and gas coupling resulting from extragalactic halo formation during ram pressure stripping needs to be undertaken on a case by case basis. We plan to do this in a future paper.

Acknowledgements. M.B. and E.M.X. wish to acknowledge Kalliopi Dasyra for the development of the code (Misiriotis et al. 2006) used to calculate the radiation field around NGC 891. We wish to thank our referee A.G.G.M. Tielens for a careful reading and many helpful remarks.

\section{Appendix A: Upgrades to DustEM}

In the DustEM model version 4.0, the only source of heating is the absorption of photons. As we have seen, in the IGM, electron collisional heating can play an important role and can be also more important than the photon heating.

In addition to the already present photon heating we implemented an electron collisional heating routine (DM_get_collheat) in the model. In this routine we evaluate the power absorbed per grain due to collisions between grains and electrons as described in Sect. 3.

The routine DM_get_collheat is then called in the routine DM_get_tdist, responsible for the calculation of the temperature distribution. This latter routine has been changed in order to take into account the electron collisional heating and the fact that the total power absorbed per grain is the sum of photon and collisional heating.

In the routine DM_get_collheat, the variable called nbrpho represents the rate of the absorbed photons multiplied by their energy. We created an equivalent variable called $n b r c o l l$ which is expressed by:

$\operatorname{nbrcoll}(E)=n_{\mathrm{e}} v(E) f_{\mathrm{M}}(E) \zeta(E) E$

where $n_{\mathrm{H}}$ is the gas number density, $v(E)$ the velocity of the impinging electrons, $f_{\mathrm{M}}(E)$ the Maxwell-Boltzmann distribution and $\zeta(E) E$ the fraction of energy deposited in the grain.

In the routine DM_get_tdist the variable hnumax represents the maximum energy that can be absorbed by a grain. If we consider only the photon absorption, the energy of the hardest photons in a $\mathrm{HI}$ region is $13.6 \mathrm{eV}$. In the case of electron collisional heating, this value can be much larger. We modified the variable numax in order to be the maximum between $13.6 \mathrm{eV}$ and the energy of the most energetic electrons.

In the routine DM_get_tdist, to make the program faster, we distinguish between stochastic heating and thermal equilibrium of dust grains. In Sect. 8 we compared the average time between two events (be they the absorption of photons or collisions with electrons) and the cooling time. When the cooling time starts to be longer than the average time between two events the grain is in a transition between stochastic heating and thermal equilibrium but it is still considered as stochastically heated by the routine DM_get_tdist. Then, when the cooling time is much longer than the average time between two events, it is considered to be in thermal equilibrium. This condition is modelled in the following way: if $\left(U_{\text {equi }}-h v_{\max }\right)>U_{\min }$ is satisfied then the grain is in thermal equilibrium. $U_{\text {equi }}$ is defined as the internal energy when the grain is in thermal equilibrium and $U_{\min }$ is the internal energy corresponding to the grain at a temperature equals to the $\mathrm{CMB}$ temperature. This is valid since the only source of heating is the photon absorption. After the addition of the collisional heating we changed this condition in this way: $\left(U_{\text {eq }}-E^{*}\right)>U_{\min }$, with $E^{*}=n \_p h o \_i n t * 13.6 \mathrm{eV}+n \_e l \_i n t * \max (\operatorname{tran} s f)$. We define:

$n \_p h o \_i n t=\frac{1}{h} \int_{0}^{\infty} n_{\mathrm{e}} v(E) f_{\mathrm{M}}(E) \zeta(E) E \mathrm{~d} E$

$n \_e l \_i n t=\frac{1}{h} \int_{v_{\min }}^{v_{\max }} Q_{\mathrm{abs}} J_{\nu} \mathrm{d} v$

and $\max (\operatorname{trans} f)$ as the maximum transferred energy due to collisional heating.

Finally, the number of points used for the characterization of the temperature distribution has been changed to 400, instead of 200, when we take into account collisional heating. This slows the program a little but allows us to derive the correct temperature distribution for the largest absorbed energies.

\section{References}

Alton, P. B., Bianchi, S., Rand, R. J., et al. 1998, ApJ, 507, L125

Berger, M. J., \& Seltzer, S. M. 1964, NASA SP, 3012

Bianchi, S. 2008, A\&A, 490, 461

Bianchi, S., \& Xilouris, E. M. 2011, A\&A, 531, L11

Bocchio, M., Micelotta, E. R., Gautier, A.-L., \& Jones, A. P. 2012, A\&A, 545, A124

Christensen, J. H. 1990, MNRAS, 246, 535

Compiègne, M., Verstraete, L., Jones, A., et al. 2011, A\&A, 525, A103

Cortese, L., Davies, J. I., Pohlen, M., et al. 2010, A\&A, 518, L49

Dasyra, K. M., Xilouris, E. M., Misiriotis, A., \& Kylafis, N. D. 2005, A\&A, 437, 447

Désert, F. X., Boulanger, F. \& Shore, S. N. 1986, A\&A, 160, 295

Draine, B. T., \& Li, A. 2007, ApJ, 657, 810

Draine, B. T., \& Salpeter, E. E. 1979, ApJ, 231, 77

Dwek, E. 1986, ApJ, 302, 363

Dwek, E. 1987, ApJ, 322, 812

Fioc, M., \& Rocca-Volmerange, B. 1997, A\&A, 326, 950

Iskef, H., Cunningham, J. W., \& Watt, D. E. 1983, Phys. Med. Biol., 28, 535

Jochims, H. W., Ruhl, E., Baumgartel, H., Tobita, S., \& Leach, S. 1994, ApJ, 420, 307

Jones, A. P. 2012, A\&A, 542, A98

Joy, D. C. 1995, Scanning, 17, 270

Mathis, J. S., Mezger, P. G., \& Panagia, N. 1983, A\&A, 128, 212

Micelotta, E. R., Jones, A. P., \& Tielens, A. G. G. M. 2010, A\&A, 510, A37

Misiriotis, A., Papadakis, I. E., Kylafis, N. D., \& Papamastorakis, J. 2004, A\&A, 417,39

Misiriotis, A., Xilouris, E. M., Papamastorakis, J., Boumis, P., \& Goudis, C. D. 2006, A\&A, 459, 113

Popescu, C. C., Misiriotis, A., Kylafis, N. D., Tuffs, R. J., \& Fischera, J. 2000, A\&A, 362, 138

Xilouris, E. M., Byun, Y. I., Kylafis, N. D., Paleologou, E. V., \& Papamastorakis, J. 1999, A\&A, 344, 868 